\title{
A LINK BETWEEN THE LOG-SOBOLEV INEQUALITY AND LYAPUNOV CONDITION
}

\author{
YUAN LIU
}

\begin{abstract}
We give an alternative look at the log-Sobolev inequality (LSI in short) for log-concave measures by semigroup tools. The similar idea yields a heat flow proof of LSI under some quadratic Lyapunov condition for symmetric diffusions on Riemannian manifolds provided the Bakry-Emery's curvature is bounded from below. Let's mention that, the general $\phi$-Lyapunov conditions were introduced by Cattiaux-Guillin-Wang-Wu 8 ] to study functional inequalities, and the above result on LSI was first proved subject to $\phi(\cdot)=d^{2}\left(\cdot, x_{0}\right)$ by Cattiaux-Guillin-Wu 9 through a combination of detective $L^{2}$ transportationinformation inequality $\mathrm{W}_{2} \mathrm{I}$ and the HWI inequality of Otto-Villani.

Next, we assert a converse implication that the Lyapunov condition can be derived from LSI, which means their equivalence in the above setting.
\end{abstract}

\section{INTRODUCTION}

Our aim of this paper is to give a direct proof of the log-Sobolev inequality (LSI for short) for symmetric diffusions under the Lyapunov condition and curvature condition, and the converse implication will be investigated further.

In the sequel, denote by $E$ a connected complete Riemannian manifold of finite dimension, $d$ the geodesic distance, $\mathrm{d} x$ the volume measure, $\mu(\mathrm{d} x)=e^{-V(x)} \mathrm{d} x$ a probability measure with $V \in C^{2}(E), \mathrm{L}=\Delta-\nabla V \cdot \nabla$ the $\mu$-symmetric diffusion operator with domain $\mathcal{D}(\mathrm{L})$ in $L^{2}(\mu), P_{t}=e^{t \mathrm{~L}}$ the semigroup, $\Gamma(f, g)=\nabla f \cdot \nabla g$ the carré du champ operator, and $\mathcal{E}(f, g)=\int \Gamma(f, g) \mathrm{d} \mu$ the Dirichlet form with domain $\mathcal{D}(\mathcal{E})$ in $L^{2}(\mu)$. It is known that the integration by parts formula reads

$$
\mathcal{E}(f, g)=-\int f \mathrm{~L} g \mathrm{~d} \mu, \quad \forall f \in \mathcal{D}(\mathcal{E}), g \in \mathcal{D}(\mathrm{L}),
$$

and $P_{t}$ is $L^{2}$-ergodic, i.e.

$$
\left\|P_{t} f-\mu f\right\|_{L^{2}(\mu)} \rightarrow 0 \text { as } t \rightarrow \infty, \forall f \in L^{2}(\mu) .
$$

We refer to Bakry-Gentil-Ledoux [5] for a detailed presentation of the fundamentals. For simplicity, write $\mu f^{2}=\int f^{2} \mathrm{~d} \mu, P_{t} f^{2}=P_{t}\left(f^{2}\right)$, and $\mathcal{E}[f]=\mathcal{E}(f, f)$.

A (tight) LSI means there exists a constant $C>0$ such that for any $f \in \mathcal{D}(\mathcal{E})$

$$
\operatorname{Ent}\left(f^{2}\right):=\mu\left(f^{2} \log f^{2}\right)-\mu f^{2} \log \mu f^{2} \leqslant C \mathcal{E}(f, f) .
$$

There have been several classical proofs of LSI for log-concave measures, such as [4, 6, 7, 12. We would like to revisit this case in another simple viewpoint.

Date: July 31, 2018.

2010 Mathematics Subject Classification. 26D10, 47D07, 60E15, 60J60.

Key words and phrases. log-Sobolev inequality, log-concave measure, heat flow, symmetric diffusion, Lyapunov condition. 
Proposition 1.1. Suppose $\mu$ is log-concave in $\mathbb{R}^{n}$ such that $\operatorname{Hess}(V) \geqslant c \mathrm{Id}$ with $c>0$. Then the LSI (1.1) holds for $C=2 / c$.

Set $\Phi(t)=2 \mathcal{E}\left[\sqrt{P_{t} f}\right]-c \operatorname{Ent}\left(P_{t} f\right)$ for $f>0$. Heuristically, due to $P_{t} f \rightarrow \mu f$ in $L^{2}$-norm, we have $\Phi(\infty)=0$ and then $\Phi(0) \geqslant 0$ if there holds the monotonicity

$$
\frac{\mathrm{d} \Phi}{\mathrm{d} t} \leqslant 0 \text {. }
$$

Actually, using the integration by parts formula and next two equalities for $\varphi=P_{t} f$

$$
\frac{\partial}{\partial t} \varphi=\mathrm{L} \varphi, \quad \nabla \mathrm{L} \varphi=\mathrm{L} \nabla \varphi-\operatorname{Hess}(V) \nabla \varphi
$$

we can calculate $\frac{\mathrm{d} \Phi}{\mathrm{d} t}$ to imply (1.2) automatically.

For general cases, some practical conditions have been presented to derive LSI. In particular, Cattiaux-Guillin-Wang-Wu 8 introduced the $\phi$-Lyapunov conditions to study super Poincaré inequalities and LSI. Cattiaux-Guillin-Wu [9] mainly discussed the "quadratic" Lyapunov condition as $\phi(\cdot)=d^{2}\left(\cdot, x_{0}\right)$ to derive the Talagrand's inequality $\mathrm{W}_{2} \mathrm{H}$, and also LSI provided that the Bakry-Emery's curvature has a lower bound (actually, they gave more than that when the curvature had a decay rate and the Lyapunov condition became stronger correspondingly). So our first aim comes out of here. Recall that the proof of LSI in [9] relied on a combination of detective $L^{2}$ transportation-information inequality $\mathrm{W}_{2} \mathrm{I}$ and the HWI inequality in Otto-Villani [13. We want to give a direct proof of this fact via the similar idea of monotonicity on heat flow as for Proposition 1.1.

With a little relaxation, say $W>0$ is a Lyapunov function if $W^{-1}$ is locally bounded and there exist two constants $c>0, b \geqslant 0$ and some $x_{0} \in E$ such that in the sense of distribution

$$
\mathrm{L} W \leqslant\left(-c d^{2}\left(x, x_{0}\right)+b\right) W .
$$

Note that we request $W>0$ other than $W \geqslant 1$ in 9 , but if $W^{-1}$ is locally bounded, the technique of Bakry-Barthe-Cattiaux-Guillin [2, Theorem 1.4] still works, which plays an important role in studying various functional inequalities via the Lyapunov type conditions. We prove that

Theorem 1.2. Suppose $\operatorname{Ric}+\operatorname{Hess}(V) \geqslant-K \operatorname{Id}$ with $K \in \mathbb{R}^{+}$. Then the LSI (1.1) holds under the Lyapunov condition (1.3).

We further investigate how to derive the Lyapunov condition from LSI.

Theorem 1.3. If the LSI (1.1) holds, there exists $W>0$ satisfying (1.3).

As consequence, it follows

Corollary 1.4. Suppose $\operatorname{Ric}+\operatorname{Hess}(V) \geqslant-K \mathrm{Id}$ with constant $K \geqslant 0$. Then the LSI (1.1) is equivalent to the Lyapunov condition (1.3).

Since the Poincaré inequality will serve as a basic tool in our proofs, it is necessary to take some related literature into account. Cattiaux-Guillin-Zitt [10] sought out the links around the Poincaré inequality, exponential contraction of diffusion semigroup, exponential integrability of hitting time, and (weak) Lyapunov condition as

$$
\mathrm{L} W \leqslant-\lambda W \text { on } \bar{U}^{c}, \text { with } W \geqslant 1, \lambda>0, U \text { open connected and bounded, }
$$


which made a continuous-time analogy with those characterizations for irreducible and aperiodic Markov chains on countable state spaces. Bakry-Cattiaux-Guillin [3] discussed also the relations between the Poincaré inequality and certain Lyapunov condition associated with a closed Petite set. Note that our argument for Corollary 1.4 can not be applied to the above quoted results.

Next two sections will be devoted to the proofs of Theorem 1.2 1.3 respectively.

\section{Proof of Theorem 1.2}

First of all, we show that the Lyapunov condition (1.3) acts the same as in 9 .

Lemma 2.1. (2. Theorem 1.4]) The Poincaré inequality holds under the Lyapunov condition (1.3). Moreover, for any $h \in \mathcal{D}(\mathcal{E})$

$$
\int h^{2}(x) d^{2}\left(x, x_{0}\right) \mathrm{d} \mu(x) \leqslant \frac{1}{c} \int|\nabla h|^{2} \mathrm{~d} \mu+\frac{b}{c} \int h^{2} \mathrm{~d} \mu .
$$

Proof. It follows from (1.3) that

$$
\mathrm{L} W \leqslant\left[-c+(c+b) \mathbf{1}_{B_{1}(0)}\right] W
$$

which implies the Poincaré inequality by the argument of [2, Page 64]. Moreover,

$$
\begin{aligned}
\int h^{2}(x) d^{2}\left(x, x_{0}\right) \mathrm{d} \mu(x) & =\frac{1}{c} \int h^{2}\left(c d^{2}\left(x, x_{0}\right)-b\right) \mathrm{d} \mu+\frac{b}{c} \int h^{2} \mathrm{~d} \mu \\
& \leqslant \frac{1}{c} \int \frac{-\mathrm{L} W}{W} h^{2} \mathrm{~d} \mu+\frac{b}{c} \int h^{2} \mathrm{~d} \mu \\
& =\frac{1}{c} \int \nabla W \cdot \nabla \frac{h^{2}}{W} \mathrm{~d} \mu+\frac{b}{c} \int h^{2} \mathrm{~d} \mu \\
& =\frac{1}{c} \int|\nabla h|^{2}-\left|\nabla h-\frac{h}{W} \nabla W\right|^{2} \mathrm{~d} \mu+\frac{b}{c} \int h^{2} \mathrm{~d} \mu \\
& \leqslant \frac{1}{c} \int|\nabla h|^{2} \mathrm{~d} \mu+\frac{b}{c} \int h^{2} \mathrm{~d} \mu .
\end{aligned}
$$

Note that here is no need to assume the integrability of $d^{2}\left(x, x_{0}\right)$ or $\frac{-\mathrm{L} W}{W}$ for $\mu$, since we can take an approximation sequence in $C_{\mathrm{c}}^{\infty}(E)$ for given $h$.

Lemma 2.2. The curvature condition $\operatorname{Ric}+\operatorname{Hess}(V) \geqslant-K$ with $K \in \mathbb{R}$ implies

(1) For any $f \in \mathcal{B}_{b}^{+}, x, y \in E$ and $t>0$

$$
\left(P_{t} f\right)^{2}(x) \leqslant P_{t} f^{2}(y) \exp \left(\frac{K d^{2}(x, y)}{1-e^{-2 K t}}\right) .
$$

(2) For any $f \in C_{\mathrm{b}}^{1}$ and $t>0$

$$
\left|\nabla P_{t} f\right| \leqslant e^{K t} P_{t}|\nabla f| \text {. }
$$

Proof. Refer to [5, Section 5.5-5.6] or Wang [15, Theorem 2.3.3].

Corollary 2.3. If $\operatorname{Ric}+\operatorname{Hess}(V) \geqslant-K$ with $K \in \mathbb{R}^{+}$, set $\mu_{0}=\mu e^{-2 K d^{2}\left(x_{0}, \cdot\right)}$, then

$$
\frac{\left(P_{t} f\right)^{2}(x)}{\mu f^{2}} \leqslant \mu_{0}^{-\frac{1}{1-e^{-2 K t}}} \exp \left(\frac{2 K}{1-e^{-2 K t}} d^{2}\left(x_{0}, x\right)\right) .
$$


Proof. Denote $\delta(t)=1-e^{-2 K t} \leqslant 1$. Using Lemma 2.2 yields

$$
\begin{aligned}
\left(P_{t} f^{2}(y)\right)^{\delta(t)} & \geqslant\left(P_{t} f\right)^{2 \delta(t)}(x) \exp \left(-K d^{2}(x, y)\right) \\
& \geqslant\left(P_{t} f\right)^{2 \delta(t)}(x) \exp \left(-2 K d^{2}\left(x_{0}, x\right)-2 K d^{2}\left(x_{0}, y\right)\right) .
\end{aligned}
$$

Integrating in $y$ on both sides gives

$$
\mu\left(\left(P_{t} f^{2}\right)^{\delta(t)}\right) \geqslant\left(P_{t} f\right)^{2 \delta(t)}(x) \exp \left(-2 K d^{2}\left(x_{0}, x\right)\right) \mu_{0},
$$

which implies (2.4) by the Hölder inequality $\mu\left(\left(P_{t} f^{2}\right)^{\delta(t)}\right) \leqslant\left(\mu\left(P_{t} f^{2}\right)\right)^{\delta(t)}$.

Now we prove Theorem 1.2 .

Proof. The strategy contains three steps. Assume $f$ is a bounded smooth function.

Step 1. Abbreviate $\varphi=P_{t} f$, we introduce

$$
\operatorname{Ent}^{*}\left(\varphi^{2}\right):=\int \varphi^{2} \log \frac{\varphi^{2}}{\mu f^{2}} \mathrm{~d} \mu,
$$

and

$$
\Psi(t):=\mathcal{E}[\varphi]+A \mu(\varphi-\mu \varphi)^{2}-\eta \operatorname{Ent}^{*}\left(\varphi^{2}\right),
$$

where $A$ and $\eta$ are two positive constants which will be decided below.

Derivative calculations give respectively

$$
\begin{aligned}
\frac{\mathrm{d}}{\mathrm{d} t} \mathcal{E}[\varphi] & =-2 \mu|\nabla \nabla \varphi|^{2}-2 \mu[(\operatorname{Ric}+\operatorname{Hess}(V))(\nabla \varphi, \nabla \varphi)] \\
A \frac{\mathrm{d}}{\mathrm{d} t} \mu(\varphi-\mu \varphi)^{2} & =-2 A \mu\left(|\nabla \varphi|^{2}\right) \\
-\eta \frac{\mathrm{d}}{\mathrm{d} t} \operatorname{Ent}^{*}\left(\varphi^{2}\right) & =2 \eta \mu\left(|\nabla \varphi|^{2} \log \frac{\varphi^{2}}{\mu f^{2}}\right)+6 \eta \mu|\nabla \varphi|^{2}
\end{aligned}
$$

Here $\nabla \nabla \varphi$ denotes the Hessian of $\varphi$. Due to the curvature condition, (2.5) is less than $-2 \mu|\nabla \nabla \varphi|^{2}+2 K \mu|\nabla \varphi|^{2}$. To estimate (2.7), using (2.4) gives

$$
\mu\left(|\nabla \varphi|^{2} \log \frac{\varphi^{2}}{\mu f^{2}}\right) \leqslant \frac{2 K}{1-e^{-2 K t}} \mu\left(|\nabla \varphi|^{2} d^{2}\left(x, x_{0}\right)\right)-\frac{\log \mu_{0}}{1-e^{-2 K t}} \mu|\nabla \varphi|^{2} .
$$

We fix $t_{0}=1$ (or any positive number) and set $\eta=c \frac{1-e^{-2 K t_{0}}}{2 K}$. Applying (2.1) to the above inequality for $h^{2}=|\nabla \varphi|^{2}$ yields for all $t \geqslant t_{0}$

$$
2 \eta \mu\left(|\nabla \varphi|^{2} \log \frac{\varphi^{2}}{\mu f^{2}}\right) \leqslant 2 \mu|\nabla \nabla \varphi|^{2}+2 b \mu|\nabla \varphi|^{2}-\frac{c \log \mu_{0}}{K} \mu|\nabla \varphi|^{2} .
$$

Set $A=K+b-\frac{c \log \mu_{0}}{2 K}+3 \eta$ (note that $\mu_{0} \leqslant 1$ ), we obtain by combining (2.5) [2.7) with (2.8)

which implies

$$
\frac{\mathrm{d}}{\mathrm{d} t} \Psi(t) \leqslant 0, \quad \forall t \geqslant t_{0}
$$

$$
\Psi\left(t_{0}\right) \geqslant \Psi(t) \geqslant \Psi(\infty) \geqslant 0, \quad \forall t \geqslant t_{0}
$$

namely

$$
\eta \operatorname{Ent}^{*}\left(\varphi^{2}\right) \leqslant \mathcal{E}[\varphi]+A \mu(\varphi-\mu \varphi)^{2}, \quad \forall t \geqslant t_{0}
$$


Step 2. For $0<t<t_{0}$, it is invalid to prove $\frac{\mathrm{d}}{\mathrm{d} t} \Psi(t) \leqslant 0$ in the above manner. Nevertheless, we turn to comparing $\operatorname{Ent}\left(P_{t_{0}} f^{2}\right)$ with Ent* $\left(\left(P_{t_{0}} f\right)^{2}\right)$ directly (and thus, it is allowed to alter the definition of $\Psi$ for $\left.0<t<t_{0}\right)$. Define

$$
\Theta_{1}(t)=\int\left(P_{t} f^{2}-\left(P_{t} f\right)^{2}\right) \log \frac{\left(P_{t} f\right)^{2}}{\mu f^{2}} \mathrm{~d} \mu, \quad \Theta_{2}(t)=\int P_{t} f^{2} \log \frac{P_{t} f^{2}}{\left(P_{t} f\right)^{2}} \mathrm{~d} \mu
$$

which satisfy

$$
\operatorname{Ent}\left(P_{t_{0}} f^{2}\right)-\operatorname{Ent}^{*}\left(\left(P_{t_{0}} f\right)^{2}\right)=\Theta_{1}\left(t_{0}\right)+\Theta_{2}\left(t_{0}\right) .
$$

Firstly, it follows from (2.4)

$$
\begin{aligned}
& \Theta_{1}\left(t_{0}\right) \\
\leqslant & \frac{2 K}{1-e^{-2 K t_{0}}} \mu\left[\left(P_{t_{0}} f^{2}-\left(P_{t_{0}} f\right)^{2}\right) d^{2}\left(x, x_{0}\right)\right]-\frac{\log \mu_{0}}{1-e^{-2 K t_{0}}} \mu\left(P_{t_{0}} f^{2}-\left(P_{t_{0}} f\right)^{2}\right) \\
\leqslant & \frac{2 K}{1-e^{-2 K t_{0}}} \mu\left(P_{t_{0}} f^{2} \cdot d^{2}\left(x, x_{0}\right)\right)-\frac{\log \mu_{0}}{1-e^{-2 K t_{0}}} \mu\left(f^{2}-(\mu f)^{2}\right) .
\end{aligned}
$$

Using (2.1), (2.3) and the Hölder inequality yields

$$
\begin{aligned}
\mu\left(P_{t_{0}} f^{2} \cdot d^{2}\left(x, x_{0}\right)\right) & \leqslant c^{-1} \mu\left|\nabla \sqrt{P_{t_{0}} f^{2}}\right|^{2}+b c^{-1} \mu f^{2} \\
& =(4 c)^{-1} \mu \frac{\left|\nabla P_{t_{0}} f^{2}\right|^{2}}{P_{t_{0}} f^{2}}+b c^{-1} \mu f^{2} \\
& \leqslant(4 c)^{-1} e^{2 K t_{0}} \mu \frac{\left(P_{t_{0}}\left|\nabla f^{2}\right|\right)^{2}}{P_{t_{0}} f^{2}}+b c^{-1} \mu f^{2} \\
& =c^{-1} e^{2 K t_{0}} \mu \frac{\left(P_{t_{0}}|f \nabla f|\right)^{2}}{P_{t_{0}} f^{2}}+b c^{-1} \mu f^{2} \\
& \leqslant c^{-1} e^{2 K t_{0}} \mu|\nabla f|^{2}+b c^{-1} \mu f^{2} .
\end{aligned}
$$

Combining the above two estimates gives

$$
\Theta_{1}\left(t_{0}\right) \leqslant C_{1} \mathcal{E}[f]+C_{2} \mu f^{2}+C_{3} \mu(f-\mu f)^{2},
$$

where $C_{1}=\frac{2 K e^{2 K t_{0}}}{c\left(1-e^{-2 K t_{0}}\right)}, C_{2}=\frac{2 b K}{c\left(1-e^{-2 K t_{0}}\right)}$ and $C_{3}=-\frac{\log \mu_{0}}{1-e^{-2 K t_{0}}}$.

Secondly, due to $\Theta_{2}(0)=0$, there is an integral representation

$$
\begin{aligned}
\Theta_{2}\left(t_{0}\right) & =\int_{0}^{t_{0}} \Theta_{2}^{\prime}(t) \mathrm{d} t \\
& =\int_{0}^{t_{0}} \int \mathrm{L} P_{t} f^{2} \log \frac{P_{t} f^{2}}{\left(P_{t} f\right)^{2}}+\mathrm{L} P_{t} f^{2}-2 \frac{P_{t} f^{2}}{P_{t} f} \mathrm{~L} P_{t} f \mathrm{~d} \mu \mathrm{d} t \\
& =\int_{0}^{t_{0}} \int \mathrm{L} P_{t} f^{2} \log \frac{P_{t} f^{2}}{\left(P_{t} f\right)^{2}}-2 \frac{P_{t} f^{2}}{P_{t} f} \mathrm{~L} P_{t} f \mathrm{~d} \mu \mathrm{d} t \\
& =\int_{0}^{t_{0}} \int-\frac{\left|\nabla P_{t} f^{2}\right|^{2}}{P_{t} f^{2}}+4 \frac{\nabla P_{t} f^{2} \cdot \nabla P_{t} f}{P_{t} f}-2 \frac{P_{t} f^{2}\left|\nabla P_{t} f\right|^{2}}{\left(P_{t} f\right)^{2}} \mathrm{~d} \mu \mathrm{d} t \\
& \leqslant \int_{0}^{t_{0}} \int \frac{\left|\nabla P_{t} f^{2}\right|^{2}}{P_{t} f^{2}}-2\left(\frac{\left|\nabla P_{t} f^{2}\right|}{\sqrt{P_{t} f^{2}}}-\frac{\sqrt{P_{t} f^{2}}\left|\nabla P_{t} f\right|}{P_{t} f}\right)^{2} \mathrm{~d} \mu \mathrm{d} t,
\end{aligned}
$$


which implies through (2.3) and the Hölder inequality

$$
\begin{aligned}
\Theta_{2}\left(t_{0}\right) & \leqslant \int_{0}^{t_{0}} \mu \frac{\left|\nabla P_{t} f^{2}\right|^{2}}{P_{t} f^{2}} \mathrm{~d} t \leqslant \int_{0}^{t_{0}} 4 e^{2 K t} \mu \frac{\left(P_{t}|f \nabla f|\right)^{2}}{P_{t} f^{2}} \mathrm{~d} t \\
& \leqslant \int_{0}^{t_{0}} 4 e^{2 K t} \mu|\nabla f|^{2} \mathrm{~d} t=: C_{4} \mathcal{E}[f],
\end{aligned}
$$

where $C_{4}=\frac{2\left(e^{2 K t_{0}}-1\right)}{K}$.

Combining (2.9) with (2.10) gives

$$
\operatorname{Ent}\left(P_{t_{0}} f^{2}\right)-\operatorname{Ent}^{*}\left(\left(P_{t_{0}} f\right)^{2}\right) \leqslant\left(C_{1}+C_{4}\right) \mathcal{E}[f]+C_{2} \mu f^{2}+C_{3} \mu(f-\mu f)^{2} .
$$

Recall the last inequality in Step 1 together with the monotonicity of $\mathcal{E}[\varphi]$ in $t$ (see [5. Proposition 3.1.6]), we obtain

$$
\eta \operatorname{Ent}\left(P_{t_{0}} f^{2}\right) \leqslant\left[1+\eta\left(C_{1}+C_{4}\right)\right] \mathcal{E}[f]+\eta C_{2} \mu f^{2}+\left(A+\eta C_{3}\right) \mu(f-\mu f)^{2} .
$$

Step 3. Now it is feasible to employ a new form of $\Psi$ for $0<t<t_{0}$ like

$$
\left(1+t_{0}-t\right)\left(A_{1} \mathcal{E}[f]+A_{2} \mu f^{2}+A_{3} \mu(f-\mu f)^{2}\right)-\eta \operatorname{Ent}\left(P_{t} f^{2}\right)
$$

to show its monotonicity further. In an equivalent and quick way, it is enough to use the same argument as (2.10) to get

$$
\begin{aligned}
\operatorname{Ent}\left(f^{2}\right)-\operatorname{Ent}\left(P_{t_{0}} f^{2}\right) & =\int_{0}^{t_{0}}-\frac{\mathrm{d}}{\mathrm{d} t} \operatorname{Ent}\left(P_{t} f^{2}\right) \mathrm{d} t \\
& =\int_{0}^{t_{0}} \int-\mathrm{L} P_{t} f^{2} \log \frac{P_{t} f^{2}}{\mu f^{2}}-\mathrm{L} P_{t} f^{2} \mathrm{~d} \mu \mathrm{d} t \\
& =\int_{0}^{t_{0}} \mu \frac{\left|\nabla P_{t} f^{2}\right|^{2}}{P_{t} f^{2}} \mathrm{~d} t \leqslant C_{4} \mathcal{E}[f] .
\end{aligned}
$$

Recall the last inequality in Step 2, it follows

$$
\eta \operatorname{Ent}\left(f^{2}\right) \leqslant\left[1+\eta\left(C_{1}+2 C_{4}\right)\right] \mathcal{E}[f]+\eta C_{2} \mu f^{2}+\left(A+\eta C_{3}\right) \mu(f-\mu f)^{2} .
$$

Denote by $\lambda_{\mu}$ the spectral gap, applying the Poincaré inequality to the above estimate yields

$$
\eta \operatorname{Ent}\left(f^{2}\right) \leqslant\left[1+\eta\left(C_{1}+2 C_{4}\right)+\lambda_{\mu}^{-1}\left(A+\eta C_{3}\right)\right] \mathcal{E}[f]+\eta C_{2} \mu f^{2} .
$$

Substituting $f$ in (2.11) to $f-\mu f$, we can bound $\eta \operatorname{Ent}\left((f-\mu f)^{2}\right)$ by some linear combination of $\mathcal{E}[f]$ and $\mu(f-\mu f)^{2}$.

On the other hand, applying the Rothaus's lemma in [14] i.e. $\operatorname{Ent}\left((f+a)^{2}\right) \leqslant$ $\operatorname{Ent}\left(f^{2}\right)+2 \mu f^{2}$ for any $a \in \mathbb{R}$ yields

$$
\operatorname{Ent}\left(f^{2}\right) \leqslant \operatorname{Ent}\left((f-\mu f)^{2}\right)+2 \mu(f-\mu f)^{2},
$$

which implies an inequality as $\eta \operatorname{Ent}\left(f^{2}\right) \leqslant B_{1} \mathcal{E}[f]+B_{2} \mu(f-\mu f)^{2}$. Combining it with the Poincaré inequality again gives

$$
\eta \operatorname{Ent}\left(f^{2}\right) \leqslant C \mathcal{E}[f]
$$

with $C=1+\eta\left(C_{1}+2 C_{4}\right)+\lambda_{\mu}^{-1}\left[A+\eta\left(2+C_{2}+C_{3}\right)\right]$. 


\section{Proof of Theorem 1.3}

The construction of Lyapunov function comes from solving an elliptic equation. For convenience, suppose a LSI holds as

$$
2 \rho \operatorname{Ent}\left(f^{2}\right) \leqslant \mathcal{E}[f] .
$$

According to the Herbst's argument in Aida-Masuda-Shigekawa 1, a LSI implies the Gaussian integrability with some $c>0$ and $x_{0} \in E$

$$
\mu e^{c d^{2}\left(x, x_{0}\right)}<\infty
$$

see also [5, Proposition 5.4.1] or [12, Section 5.1].

Denote $\phi(x)=\rho\left(-c d^{2}\left(x, x_{0}\right)+b\right)$ with $b=2 \mu e^{c d^{2}\left(x, x_{0}\right)}$. We introduce the equation for $f \in L^{2}(\mu)$

$$
\mathrm{H} u:=-\mathrm{L} u+\phi u=f .
$$

Our aim is to find a continuous positive solution $u$ to (3.1) for $f \equiv 1$, which fulfills the definition of Lyapunov function and thus prove Theorem 1.3 .

Proof. The strategy contains two steps.

Step 1. Equation (3.1) gives $\mu(u \cdot \mathrm{H} u) \leqslant \mathcal{E}[u]+\rho b \mu u^{2}$ quickly. On the other hand, using the Young's inequality and LSI yields

$$
\begin{aligned}
\mu(u \cdot \mathrm{H} u) & =\mathcal{E}[u]+\rho b \mu u^{2}-\rho \mu\left(c d^{2}\left(x, x_{0}\right) u^{2}\right) \\
& \geqslant \mathcal{E}[u]+\rho b \mu u^{2}-\rho \cdot \mu u^{2} \cdot \mu\left(e^{c d^{2}\left(x, x_{0}\right)}-\frac{u^{2}}{\mu u^{2}}+\frac{u^{2}}{\mu u^{2}} \log \frac{u^{2}}{\mu u^{2}}\right) \\
& =\mathcal{E}[u]+\rho b \mu u^{2}-\frac{b}{2} \rho \mu u^{2}+\rho \mu u^{2}-\rho \operatorname{Ent}\left(u^{2}\right) \geqslant \frac{1}{2}\left(\mathcal{E}[u]+\rho b \mu u^{2}\right) .
\end{aligned}
$$

Then $\mu(u \cdot \mathrm{H} u)$ determines a coercive Dirichlet form, and $\mathrm{H}$ is a positive definite selfadjoint Schrödinger operator with its spectrum contained in $(0, \infty)$. It means $\mathrm{H}^{-1}$ exists on $L^{2}(\mu)$ according to the Lax-Milgram Theorem, i.e. $u=\mathrm{H}^{-1} f \in H^{1}(\mu)$ (i.e. the $L^{2}$-integrable Sobolev space of weak derivatives of first order) is a weak solution to Equation (3.1).

Whenever $f \geqslant 0$, the weak maximum principle yields $u=\mathrm{H}^{-1} f \geqslant 0 \mu$-a.e. too. As a routine, we set $u_{-}=-\min \{u, 0\}$, which has weak derivatives and satisfies

$$
\mu\left(u_{-} f\right)=\mu\left(u_{-} \cdot \mathrm{H} u\right)=-\mathcal{E}\left[u_{-}\right]-\mu\left(\phi u_{-}^{2}\right) \leqslant-\frac{1}{2}\left\{\mathcal{E}\left[u_{-}\right]+\rho b \mu\left(u_{-}^{2}\right)\right\} \leqslant 0 .
$$

It follows $u_{-}=0 \mu$-a.e. and thus $u \geqslant 0 \mu$-a.e.

Step 2. Now fix $f \equiv 1$. By [11, Theorem 8.22] and the notation therein, $u$ is locally Hölder continuous if we set $f^{i}=0, g=-f$ and $L=\mathrm{L}-\phi$ such that $L u=g$.

Moreover, we prove that $u>0$ everywhere. By contradiction, assume $u(y)=0$ at some $y$. Choose $r>0$ and $w \in C^{2}(E)$ arbitrarily to satisfy

$$
w \geqslant 0, w(y)>0, w_{\mid B_{r}(y)^{c}}=0 .
$$

Since $\mathrm{H} w$ is a bounded function, we can take a factor $\lambda>0$ and $v=\lambda w$ such that

$$
\mathrm{H} v=\lambda \mathrm{H} w \leqslant \frac{1}{2} .
$$

It follows on $E$

$$
\mathrm{H}(u-v)=f-\mathrm{H} v \geqslant \frac{1}{2},
$$


which implies $u-v \geqslant 0 \mu$-a.e. by the weak maximum principle. Then $u(y) \geqslant v(y)$ by the continuity, which is absurd.

As consequence, we obtain $u \in H^{1}(\mu) \cap C(E)$ is strictly positive everywhere, and $u^{-1}$ is locally bounded. So $u$ is a Lyapunov function for $\mathrm{L} u \leqslant \phi u$.

Acknowledgements. It is my great pleasure to thank Prof. Li-Ming Wu for some helpful conversations, and also thank the anonymous referee for his/her careful reading of the first version and pointing out some mistakes. This work is supported by NSFC (no. 11201456, and no. 1143000182), CAS (no. Y129161ZZ1), and Key Laboratory of Random Complex Structures and Data, Academy of Mathematics and Systems Science, Chinese Academy of Sciences (No. 2008DP173182).

\section{REFERENCES}

[1] S. Aida, T. Masuda, and I. Shigekawa. Logarithmic Sobolev inequalities and Exponential integrability, J. Funct. Anal. 126 (1994), 83-101.

[2] D. Bakry, F. Barthe, P. Cattiaux, and A. Guillin. A simple proof of the Poincaré inequality for a large class of probability measures including the log-concave case, Electron. Commun. Probab. 13 (2008), 60-66.

[3] D. Bakry, P. Cattiaux, and A. Guillin. Rates of convergence for ergodic continuous Markov processes: Lyapunov versus Poincaré, J. Funct. Anal. 254 (2008), no. (3), 727-759.

[4] D. Bakry, M. Emery. Diffusions hypercontractives. Séminaire de probabilités, XIX, 1983/84, 177-206, Lecture Notes in Math., 1123, Springer, Berlin, 1985.

[5] D. Bakry, I. Gentil, and M. Ledoux. Analysis and geometry of Markov diffusion operators. Springer, Cham, 2014.

[6] S. G. Bobkov, M. Ledoux. From Brunn-Minkowski to Brascamp-Lieb and to logarithmic Sobolev inequalities, Geom. Funct. Anal. 10 (2000), no. 5, 1028-1052.

[7] M. Capitaine, E. P. Hsu, and M. Ledoux. Martingale representation and a simple proof of logarithmic Sobolev inequalities on path spaces. Electron. Comm. Probab. 2 (1997), 71-81.

[8] P. Cattiaux, A. Guillin, F.-Y. Wang, L.-M. Wu. Lyapunov conditions for super Poincaré inequalities. J. Funct. Anal. 256 (2009), no. 6, 1821-1841.

[9] P. Cattiaux, A. Guillin, and L.-M. Wu. A note on Talagrand's transportation inequality and logarithmic Sobolev inequality, Proba. Theory Relat. Fields, 148 (2010), no. 1-2, 285-304.

[10] P. Cattiaux, A. Guillin, and P.-A. Zitt. Poincaré inequalities and hitting times. Ann. Inst. Henri Poincare Probab. Stat. 49 (2013), no. 1, 95-118.

[11] D. Gilbarg, N. Trudinger. Elliptic partial differential equations of second order. Second edition. Springer-Verlag, Berlin, 1983.

[12] M. Ledoux. The concentration of measure phenomenon. Mathematical Surveys and Monographs, 89. AMS, Providence, RI, 2001.

[13] F. Otto, C. Villani. Generalization of an inequality by Talagrand and links with the logarithmic Sobolev inequality, J. Funct. Anal. 173 (2000), no. 2, 361-400.

[14] O. S. Rothaus. Hypercontractivity and the Bakry-Emery criterion for compact Lie groups, J. Funct. Anal. 65 (1986), no. 3, 358-367.

[15] F.-Y. Wang. Analysis for diffusion processes on Riemannian manifolds. Advanced Series on Statistical Science \& Applied Probability, 18. World Scientific Publishing Co. Pte. Ltd., Hackensack, NJ, 2014.

Yuan liU, Institute of Applied Mathematics, Academy of Mathematics and Systems Science, Chinese Academy of Sciences, Beijing 100190, China

E-mail address: liuyuan@amss.ac.cn 